\title{
Distant Hybridization and Molecular Breeding
}

O hina is a big country with a huge population. To grow enough food to feed the people. generations of Chinese scientists have devoted to breed high-rield varieties of wheat and rice. two of the staple crops in China. To breed elite crops with advantageous traits of. e.g.. high yields, high quality and high stress tolerance, scientists mainly resort to two methods. distant hybridization and molecular breeding.

Distant hybridization generally refers to the cross between distantly related species or genera. It offers a way to combine the advantageous traits from the parents, and to break the boundaries of species and expand the gene pool of crops.

After more than 50 vears of unremitting efforts. scientists at the CAS Institute of Genetics and Developmental Biology (ICDB) have bred a set of new wheat varieties named after Xiaoy an through a systematic distant hybridization. Viaoran-6, one of the most important parent sources from the set gave rise to more than 80 new wheat varieties. For example. a derived variety named Kenong 199 has become one of the main planted wheat varieties in Huanghuai area since its approval in 2006.

Besides. scientists at ICDB have also made many breakthroughs in elite crops breeding using molecular breeding. which combines the edges from the fields of genomics. computational biology. systems biology. synthetic biology. etc.

The crop traits. including yields, taste quality and tolerance to environmental stresses, are usually linked to their responsive quantitative trait loci (QTLs) within the genomes of a population of organisms. Guided by the increased understanding of these links in rice and wheat. scientists at IGDB are now able to put multiple desired traits together to make new elite varieties through a carefully-designed strategy involving years of crossing. selecting and backcrossing procedures. Molecular-aided rational design, an efficient way to assembling multiple complex traits into a single variety. has proven a powerful strategy to tackle the challenges of future crop breeding and may provide a solution to feed the ever-increasing population. Now. China is a global leading member in the study of modern breeding theory and breeding of a new generation of elite crop varieties.

Scientists at ICDB have won the Outstanding Scientific and Technological Achievement Award of the Chinese Academy of Sciences in 2013 and the First Prize of the National Natural Science Award in 2017.

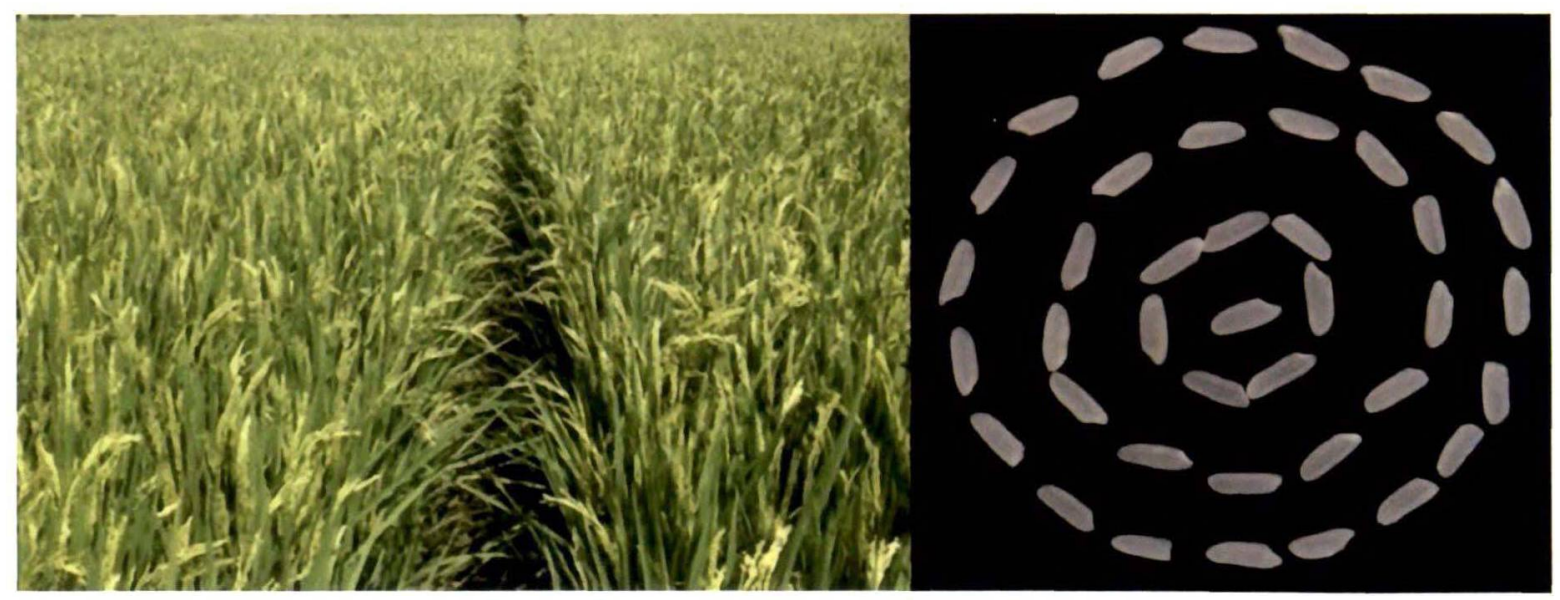

One representative elite rice variety named "Zhongke 804". (Credit: IGDB) 